\title{
MHD THERMAL INSTABILITIES IN COOL INHOMOGENEOUS ATMOSPHERES
}

\author{
G. Bodo ${ }^{1}$, A. Ferrari 2,3, S. Massaglia ${ }^{3}$ and R. Rosner ${ }^{4}$ \\ 1 Osservatorio Astronomico di Torino \\ 2 Istituto di Cosmo-geofisica del C.N.R., Torino \\ 3 Istituto di Fisica Generale, Universita' di Torino \\ 4 Harvard-Smithsonian Center for Astrophysics,Cambridge, MA, USA
}

\section{THE MODEL}

The Einstein Observatory survey of stellar coronae (Vaiana et al. 1981) and, specifically, the results on cool, low luminosity stars has suggested a correlation between stellar X-ray luminosity and stellar rotational velocity (Pallavicini et al. 1982, Walter 1981, Vaiana et al. 1981). In addition the Skylab observations of the solar corona have demonstrated a tight correlation between photospheric surface magnetic stnuctures, which emerge from the interior in the form of "loops" above the photosphere by, viz., buoyancy instabilities, (Parker 1979; see also Acheson 1979, Schmitt \& Rosner 1982, and references therein), and coronal X-ray emission (Golub et al. 1980). It therefore becomes important to ask how a coronal state (i.e. low density and high temperature plasma) of a stellar atmosphere is formed, presumably from a pure radiative equilibrium configuration.

In principle, a corona may be thought of as a metastable configuration evolved from a cool equilibrium due to some instability in which magnetic fields and currents play a basic role (Ferrari, Rosner \& Vaiana 1982). For example, these authors have shown that a plane parallel, homogeneous, infinite atmosphere in radiative equilibrium threaded by (re-entrant) magnetic lines, is unstable to MHD thermal instabilities excited by photospheric motions which produce field aligned electric currents. The heating process is related to dissipation of these currents in the presence of filamentation effects; the resulting sharp inhomogeneities lead to quasi-stationary heating processes, such as tearing mode reconnection, surface wave dissipation, fast mode damping, which were presumed to ultimately limit the filamentation process by enhancing the current diffusion rate. However, the linear instability analysis in the form presented by Ferrari, Rosner and Vaiana employed a number of simplifying approximations; one of these, namely the spatial uniformity of plasma parameters and concomitant infinite extension of magnetic structures, is relaxed in the present work.

We start from a cool plasma configuration in radiative and hydrostatic equilibrium, and assume that buoyancy instabilities have brought magnetic loops to the solar surface whose spatial scales are large compared to the instability scales considered here, and which remain "anchored" to the photosphere (see Fig. 1). We consider an initially isothermal atmosphere, whose density and pressure have scale heights fixed by the hydrostatic equations. 
Linear instability calculations, using the system of equations of motion of Ferrari, Rosner and Vaiana (1982), applied to a non-homogeneous configuration, do not yield simple analytical dispersion relations. Therefore, we have developed a numerical code which allows us to solve for oscillatory perturbations as eigenfunctions of a boundary value problem. With this code we have investigated the effects of atmospheric gradients and finite loop dimension on the scale of unstable perturbations. We want to caution that we have considered gradients for the initial equilibrium atmosphere in the upward direction (i.e. along B) only; no gradients are assumed across magnetic lines, i.e. parallel to the stellar surface. The mathematical problem is hence one-dimensional as far as eigenfunctions are concerned.

\section{THE RESULTS}

The most relevant mode for our problem is the Joule mode (Heyvaerts 1974, Ferrari et al. 1982). This mode is essentially transverse to the magnetic field, and is connected with current filamentation; the complete dispersion relation can be found in Ferrari et al. (1982). In the low frequency limit, such that the plasma can rapidly diffuse across the filaments (so that pressure build-up that could stabilize the mode can be avoided), $\omega \ll \mathrm{c}^{2} \mathrm{k}^{2} / 4 \pi \sigma_{0}$, and

$$
\omega=-\mathrm{i}(\gamma-1)\left[-\left(\kappa_{f l} \mathrm{k}_{l \mid}^{2}+\kappa_{\perp} \mathrm{k}_{\perp}^{2}\right) \mathrm{T}_{\mathrm{o}} / \mathrm{p}_{\mathrm{o}}+(\mathrm{dh} / \mathrm{dT})_{\mathrm{o}} \mathrm{T}_{\mathrm{o}} / \mathrm{p}_{\mathrm{o}}+\mathrm{J}_{\mathrm{o}}^{2}(\mathrm{~d} \ln \sigma / \mathrm{d} \ln \mathrm{T})_{\mathrm{o}}\left(\sin ^{2} \theta-\cos ^{2} \theta\right) / \sigma_{0} \mathrm{p}_{\mathrm{o}}\right.
$$

where $\kappa_{\|}$and $\kappa_{\perp}$ are the thermal parallel and perpendicular conductivities, $h=H-E_{R}$, $\theta$ is the angle between $\mathrm{k}$ and $\mathrm{B}_{\mathrm{o}}$ and the subscript "o" denotes parameters evaluated in the equilibrium state. For instability, Im $\omega<0$, which requires that the last term be positive and exceed the two preceding ones in absolute value.

In Fig. 2, we plot the dispersion relation of Joule modes in the $\left(\operatorname{Im} \omega, \mathrm{k}_{\mathrm{x}}\right)$ plane. The physical parameters used in this example apply to a "classical" stellar atmosphere, initially without any cromosphere or corona. Joule dissipation is energetically dominant in the optically thin region of the atmosphere, where $\tau \ll 1$ and $\beta \ll 1$.

As Fig. 2 shows, we find that Joule mode instability is an efficient mechanism for current filamentation and subsequent heating in initially cool atmospheres. In particular, this instability is mainly effective at the top of magnetic loops and is not suppressed by thermal conduction.

Typical scales of current filaments ( $\mathrm{L}=10 \div 10^{3} \mathrm{~cm}$ for the case of Fig. 2) are too small to be detected by currently available direct observations. However, they are sufficiently small to satisfy the theoretical requirements of steady creation of current inhomogeneities and steep magnetic gradients imposed, but generally left unexplained, in many coronal heating models.

Acknowledgments. This work was supported by NASA under the Solar Terrestrial Theory Program grant NAGW-79 to the Harvard College Observatory (RR) and by the Servizio Attivita' Spaziali del CNR (GB, AF and SM).

Acheson, D.J. 1979 Solar Physics, 62, p.23

Ferrari, A., Rosner, R., and Vaiana, G. S. 1982, Ap. J. in press

Golub, L., et al. 1980, Ap. J., 238 , p. 343

Heyvaerts, J. 1974, Astron. Astrphys. 37, p. 65 
Pallavicini, R., et al. 1982, in $2^{\text {nd }}$ Cambridge Cool Stars Workshop, ed. L. Golub and M. Giampapa

Parker, E. N. 1979, Cosmical Magnetic Fields (Oxford, Clarendon Press)

Schmitt, J. and Rosner, R. 1982, Ap. J. in press

Vaiana, G. S. et al. 1981 , Ap. J. 244, p. 163

Walter, F. G.. 1981, Ap. J. 245, p. 677

Figure 1 - Model atmosphere:

a) realistic configuration,

b) model configuration.

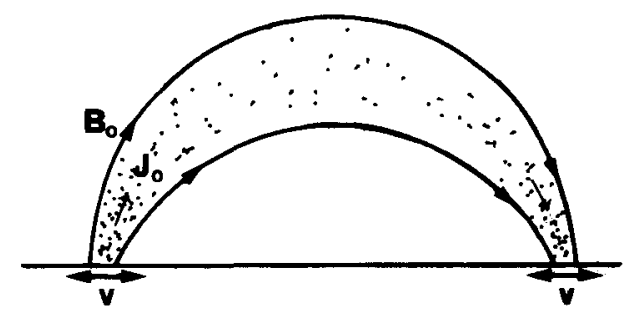

a)
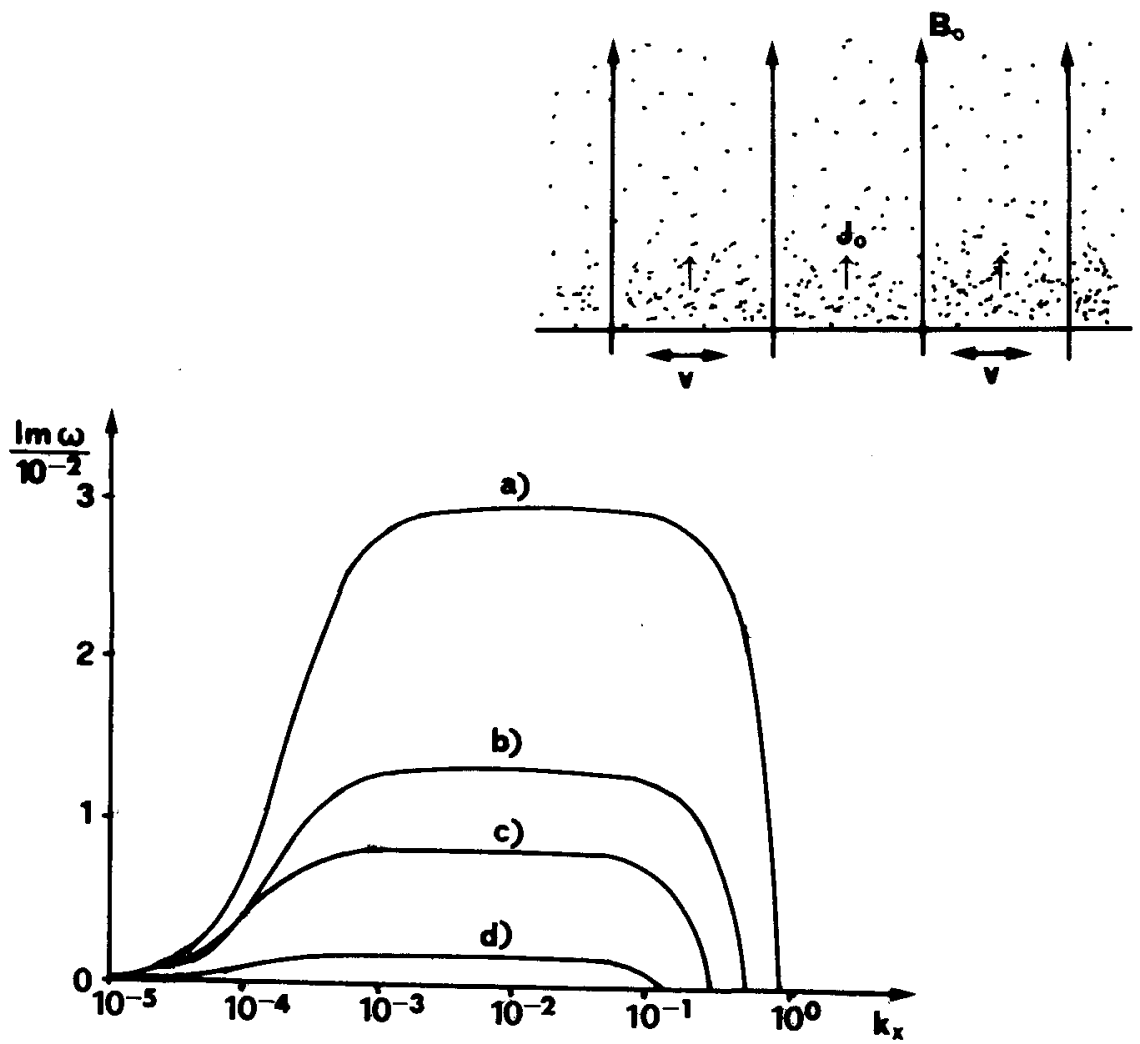

Figure $2-a)$ and c): analytic solutions of the homogeneous, infinite atmosphere $\left(\mathrm{k}_{11} \rightarrow 0\right)$ dispersion relation; a) refers to plasma conditions at the top of the loop, $\mathrm{n}=2.9 \times 10^{6} \mathrm{~cm}^{-3}$, c) to the bottom of the loop, $\mathrm{n}=10^{7} \mathrm{~cm}^{-3}$. b): dispersion relation for the case of a model atmosphere with longitudinal gradients.

d): growth rate for a homogeneous atmosphere, in which the loop has finite dimensions, and boundary conditions become important in defining the eigenfunction. 